\title{
MODELO PARA GESTIONAR EL CONOCIMIENTO EN EL SECTOR TEXTIL DE MEDELLÍN, EMPLEANDO DINÁMICA DE SISTEMAS*
}

Recibido: 22 de septiembre de 2015 • Aprobado: 20 de noviembre de 2015

\author{
Natalia Marulanda Grisales** \\ Iván A. Montoya Restrepo ${ }^{* * *}$
}

\section{RESUMEN}

Este artículo evalúa la relación de causalidad entre la gestión del conocimiento y las capacidades de innovación tecnológica, y el efecto de esta relación sobre los resultados operacionales del sector textil en la ciudad de Medellín. Se empleó la metodología de dinámica de sistemas, con simulación de escenarios para valorar las condiciones actuales de las organizaciones del sector en términos de acumulación de conocimiento y capacidades. La información se obtuvo mediante entrevistas a expertos y acceso a información especializada del sector. Se evidencia que una mejora de la relación entre la gestión del conocimiento e innovación tecnológica genera un incremento aproximado del 15\% en los ingresos operacionales del sector. Asimismo, se encontró que a medida que las variables comunes de interés (Estrategias organizacionales, canales de comunicación, formación, cultura, acciones de fortalecimiento en I+D), se acercan a los valores deseados, la acumulación de conocimiento y de capacidades de innovación tecnológica alcanzan los valores objetivos.

\section{PALABRAS CLAVE}

Capacidades de innovación tecnológica, gestión de conocimiento, recursos.

\section{CLASIFICACIÓN JEL}

C61, D21, L6, L67, O32

\section{CONTENIDO}

Introducción; 1. Sector textil; 2. Gestión de conocimiento; 3. Recursos y capacidades; 4. Metodología; 5. Resultados; 6. Conclusiones; Bibliografía.

- Artículo derivado del Trabajo Final de Maestría en Ingeniería Industrial titulado "Modelo de gestión de conocimiento en el sector textil en la ciudad de Medellín empleando dinámica de sistemas", el cual fue desarrollado por Natalia Marulanda Grisales, bajo la dirección del PhD Iván A. Montoya Restrepo del Departamento de Ingeniería de la Organización, Universidad Nacional de Colombia, Medellín. Periodo de ejecución: 2013-2015.

•* Ingeniera Industrial de la Universidad Nacional de Colombia, Medellín, Colombia. Magister en Ingeniería Universidad Nacional de Colombia, Medellín, Colombia. Docente e Investigador, Corporación Universitaria Minuto de Dios (UNIMINUTO), Bello, Colombia. Dirección: Diagonal 75 C \# 2A-146, Int. 107. Correo electrónico:nmarulandagr@unal.edu.co.

** Administrador de empresas, Universidad Nacional de Colombia, Bogotá, Colombia. Magister en Adminis tración, Universidad Nacional de Colombia, Bogotá Colombia. Doctor en Ciencias Económicas, Universidad Nacional de Colombia, Bogotá, Colombia. Profesional Asociado, Departamento de Ingeniería de la Administración, Universidad Nacional de Colombia, Medellín, Colombia. Delegado del Concejo Superior Universitario al Concejo Directivo de la Facultad de Ciencias Agrarias, Universidad Nacional de Colombia, Medellín, Colombia. Dirección: Carrera 80 No 65-223, Bloque M8-B, Medellín, Colombia. Correo electrónico: iamonyoyar@unal.edu.co. 


\section{KNOWLEDGE MANAGEMENT MODEL FOR THE TEXTILE SECTOR IN MEDELLIN, EMPLOYING SYSTEM DYNAMICS}

\section{ABSTRACT}

This paper analyses the causal relation between knowledge management and technological innovation capacities and the effect of this relation over the operational results of the textile sector for the city of Medellin. In order to value the current state of the organizations in the sector in terms of knowledge accumulation and capacities, a dynamic system methodology with scenario simulation for evaluating the actual conditions was used. The information was obtained through interviews with experts and accessing specialized information on the sector. It is evidenced that an improvement in the relation between knowledge management and technological innovation generates and approximated increase of $15 \%$ over the operational revenue of the sector. Likewise, it was found that in the measure that common variables of interest (organizational strategies, communication channels, training, culture, actions for improving I +

D) get closer to the desired values, the knowledge accumulation and the technical innovation capacities reach their goal values.

\section{KEY WORDS}

Technological innovation capacities, knowledge management, resources.

\section{JEL CLASSIFICATION}

C61, D21, L6, L67, O32

\section{CONTENT}

Introduction; 1. Textile sector; 2. Knowledge management; 3. Resources and capacities; 4. Methodology;

5. Results; 6. Conclusions; Bibliography.

\section{MODELO PARA GERIR O CONHECIMENTO NO SECTOR TÊXTIL DE MEDELLÍN, EMPREGANDO DINÂMICA DE SISTEMAS RESUMO}

Este artigo avalia a relação de causalidade entre a gestão do conhecimento e as capacidades de inovação tecnológica, e efeito desta relação sobre os resultados operacionais do setor têxtil na cidade de Medellín. Se empregou a metodologia de dinâmica de sistemas, com simulação de cenários para valorar as condições atuais das organizações do setor em termos de acumulação de conhecimento e capacidades. A informação se obteve mediante entrevistas a expertos e acesso a informação especializada do setor. Se evidencia que uma melhora da relação entre a gestão do conhecimento e inovação tecnológica gera um incremento aproximado de 15\% nos ingressos operacionais do setor. Assim mesmo, se encontrou que a medida que as variáveis comuns de interesses (Estratégias organizacionais, canais de comunicação, formação, cultura, ações de fortalecimento em I+D), se aproximam aos valores desejados, a acumulação de conhecimento e de capacidades de inovação tecnológica alcançam os valores objetivos.

\section{PALAVRAS CHAVE}

Capacidades de inovação tecnológica, gestão de conhecimento, recursos

\section{CLASSIFICAÇÃO JEL}

C61, D21, L6, L67, 032

\section{CONTEÚDO}

Introdução; 1. Sector têxtil; 2. Gestão de conhecimento; 3. Recursos e capacidades; 4. Metodologia; 5. Resultados; 6. Conclusões; Bibliografia. 


\section{INTRODUCCIÓN}

Las condiciones económicas y las características actuales de los mercados nacionales e internacionales han contribuido con el desarrollo de procesos acelerados de innovación, los cuales les permiten a las organizaciones ser más competitivas. Para Sánchez (2007), algunas de las estrategias y metodologías de gestión tradicionales que han sido abordadas en las compañías suelen quedarse cortas con respecto a los resultados operacionales que se esperan al ser implementadas. Además, las peculiaridades propias de cada empresa como los recursos y capacidades pueden llegar a restringir la implementación de dichas metodologías.

Ahora bien, en Colombia existen algunos sectores que se destacan por apalancar el PIB nacional y las economías locales. Tal es el caso del sector textil en la ciudad de Medellín, el cual se ha encargado de dinamizar la estructura empresarial de la ciudad, lo que se ha derivado en gran medida de la realización de ferias relevantes en el ámbito internacional como lo son Colombiamoda y ColombiaTex de las Américas, según lo expresado por Vargas (2013). Por ende, las compañías requieren de herramientas de gestión capaces de adaptarse a las necesidades, recursos y capacidades que cada una posee.

Entre estas herramientas de encuentra la gestión de conocimiento (KM), la cual permite el flujo de datos, información y conocimiento entre todos los miembros y áreas funcionales de las empresas. Lo anterior facilita la creatividad de las personas, los procesos de innovación, la gestión documental y el cubrimiento de las necesidades de los clientes internos y externos. De esta manera, la habilidad de una empresa para gestionar el conocimiento se convierte en un enlace entre las características del mercado y la toma de decisiones en los procesos de innovación como es mencionado por Marulanda (2015).

El propósito de este artículo consiste en evaluar las relaciones causales existentes entre las variables y factores de la gestión del conocimiento, capacidades de innovación tecnológica y resultados operacionales del sector textil de la ciudad de Medellín. Lo anterior permite desarrollar un modelo de simulación con base en la metodología de dinámica de sistemas y análisis de escenarios, a fin de evaluar diversas alternativas para el desarrollo y potenciamiento del sector en los mercados nacionales e internacionales. Para obtener la información se recurrió al análisis bibliográfico, estudios especializados en el área y entrevistas a expertos del sector, las cuáles se realizaron de manera presencial o virtual mediante la aplicación de formularios de Google Drive.

Al considerar las diversas alternativas presentadas en el análisis de escenarios, se lograron incrementos de hasta el 15\% en los ingresos operacionales del sector. 
Si bien, el desarrollo de las variables comunes de interés favorece la acumulación de conocimiento y de capacidades de innovación tecnológica, estas características sólo se presentan bajo escenarios optimistas de simulación, pues existen barreras para su ejecución en las compañías del sector textil en la ciudad de Medellín.

Este artículo está dividido en siete secciones que se encuentran organizadas de la siguiente manera. En primer lugar, se describe las características del sector textil en Colombia y la ciudad de Medellín, al igual que las condiciones actuales en términos de innovación. En las siguientes dos secciones, se presenta la descripción conceptual de gestión de conocimiento, recursos, capacidades y capacidades de innovación tecnológica. Posteriormente, se aborda la metodología, la cual se basa en el uso de dinámica de sistemas. Para concluir, en las últimas dos secciones se presenta los resultados de la investigación, los cuales se basan en el análisis de escenarios de simulación, y en la sección final se presenta las conclusiones.

\section{SECTOR TEXTIL}

Según INEXMODA (2011a), en Colombia el sector textil representa el 10,4\% del PIB Nacional y el 10,7\% del PIB manufacturero. El sector se encuentra conformado por diferentes representantes estratégicos, que le permiten categorizarse como uno de los principales componentes de la economía nacional, así es mencionado por Vélez y otros (2013), entre los cuales se encuentran proveedores, textileros, confecciónistas y comercializadores. En este sector, se presentan algunos obstáculos que restringen el desarrollo como son: infraestructura de producción, tamaño organizacional, retención del talento humano y habilidades de innovación, tal y como dice el MCIT (2009).

Ahora bien, tal y como dice Vargas (2013), la capacidad del sector para realizar cambios rápidos y eficaces, se basa en metodologías de gestión y planeación cortoplacistas. Así pues, las características del sector se conservan en los conglomerados organizacionales de las diferentes ciudades del país. El interés de la presente investigación se centra en las empresas dedicadas a la moda, diseño y confección ubicadas en la ciudad de Medellín cuyas particularidades serán presentadas en la siguiente sección.

\subsection{Sector textil en la ciudad de Medellín}

Las compañías del sector textil en la ciudad de Medellín han logrado posicionarse en los mercados nacionales e internacionales, baso en el papel que han desempeñado los productos en ColombiaTex de las Américas y Colombiamoda. Al respecto, Vargas (2013) expresa que dichas ferias han propiciado un aumento de 
más de 70 millones de dólares en transacciones en los últimos años. Al mismo tiempo para INEXMODA (2011), la composición empresarial por eslabón de la cadena productiva del sector textil en la ciudad de Medellín se centra en el comercial -distribución.

De esta manera, los directivos del sector deben centrar sus esfuerzos en la caracterización de las necesidades del consumidor, al igual que del establecimiento de oportunidades de mejora. Entre estas se encuentran la definición de periodos adecuados de planeación, instalaciones de manufactura, y transferencia tecnológica a partir de la adquisición de franquicias y maquilas. Asimismo, es fundamental establecer alianzas estratégicas con las organizaciones líderes en el ámbito mundial como lo expresa Marulanda (2015).

\subsection{Sector textil e innovación}

En las compañías del sector textil, los desarrollos innovadores en términos tecnológicos, manufactureros y de servicios, deben ir acompañados por procesos de innovación en el ámbito comercial y distribución como menciona Vargas (2013). Además, Betancur y Vargas (2012) advierten que es un sector dinámico que se renueva con base en las características gerenciales, tecnológicas y de recurso humano. De modo similar, la innovación se encuentra relacionada con el crecimiento de los beneficios monetarios a través de la inversión en modelos de manufactura eficaces y eficientes, como mencionan Mejía, Montoya y Bravo (2011). Por ende, los adelantos más sobresalientes en el sector se basan en una oferta de bienes de valor y características especiales para los clientes.

A partir de las peculiaridades que presenta el sector, es de gran interés para los directivos de sus organizaciones encontrar metodologías y herramientas que les permita atender de manera adecuada los requerimientos del mercado, sin poner en riesgo sus recursos y el desarrollo de sus capacidades. Por ende, en el siguiente apartado se exhibe la gestión de conocimiento, como una metodología que facilita la interacción entre todas las áreas empresariales y los individuos, de tal manera que se logre mejorar la posición competitiva de las empresas.

\section{GESTIÓN DE CONOCIMIENTO}

La comprensión de la metodología de gestión del conocimiento requiere de la definición previa de algunos términos como lo son conocimiento tácito y explícito. Según Sternberg (2000) el conocimiento tácito se asimila durante la ejecución de las labores cotidianas. Para Howells (1996) dicho conocimiento hace parte de 
la habilidad individual para aprender de la experiencia, no está estructurado, se caracteriza por el know-how y no implica la generación de procesos tangibles. Al respecto, Fleck (1996) establece que el conocimiento tácito se expresa a través de los procesos de aprendizaje, entrenamiento y learning by doing. Además, como recomienda Gourlay (2006), este no necesariamente se transforma en conocimiento explícito.

Shao, Feng y Liu (2012) y Delen y otros (2013), advierten que el conocimiento explícito es preciso y metódico, puede ser recolectado, documentado y almacenado de manera sencilla. Según Polanyi (1966), este tipo de conocimiento puede transmitirse, comprenderse y compartirse sin la presencia física de un individuo. Al respecto Arnett y Wittmann (2014), admiten que la normalización y difusión del conocimiento tácito recae en los objetivos que se han planteado los directivos, pues dicho proceso no puede ser ejecutado si no se cuenta con una directriz específica por parte de los superiores.

Con todo esto, numerosos autores han desarrollado modelos que permiten coordinar ambas categorías de conocimiento, para perfeccionar los resultados operacionales. A continuación, se exhibirán los modelos que mayor acogida han tenido en términos empresariales.

\subsection{Modelos de gestión de conocimiento}

Uno de los modelos de gestión de conocimiento más aceptados es el modelo SECI (Socialización, Exteriorización, Combinación e Interiorización) propuesto por Nonaka y Takeuchi (1995). El modelo posee cuatro fases de conversión de conocimiento, las cuales son socialización, exteriorización, combinación e internalización. Sin embargo, ha contado con varias críticas con respecto a las variables empleadas, metodología, demostración de procesos y ambigüedad de conceptos, como lo mencionan Andreeva y Ikhilchik (2011), Martin y Root (2009), Gourlay (2003), Sundaresan y Zhang (2012).

Como respuesta a estas críticas se han planteado otros modelos. Por ejemplo, Zhang y Kosaka (2013) proponen el modelo KIKI (Knowledge sharing in collaboration, Identificaction of the service field, Knowledge creation for the new service idea, Implementation of the new service idea). Hedlund (1994) diseñó el modelo de Transferencia y Creación de Conocimiento. Tejedor y Aguirre (1998) desarrollaron el modelo del KPMG Consulting Group. Drucker (1988) ideó la Empresa Basada en la Información.

No obstante, como el objeto de estudio involucra etapas adicionales al proceso de creación de conocimiento, se emplearán dos macrofases Marulanda (2015): ex- 
ploración y explotación, las cuales han sido abordas por Grant (2002) y Manzanares y Gómez (2008). Dichas fases repercuten de manera directa en los procesos de innovación organizacional y en los resultados esperados del desarrollo de nuevos productos y procesos, mediante el fortalecimiento de los recursos y capacidades organizacionales.

\section{RECURSOS Y CAPACIDADES}

Según Wernerfelt (1984), Penrose (1959) y Barney (1991) los recursos son elementos propios o externos a las compañías, los cuales deben ser valiosos, raros, inimitables y no poseer sustitutos, para poder ser transformados en fortalezas de las firmas. Pueden ser tangibles o intangibles, físicos, humanos y organizacionales, como lo expresan Barney (1991), Marino (1996), Hafeez, Zhang y Malak (2002). No obstante, no todos los recursos son beneficiosos y valiosos para las organizaciones, como argumenta Grant (1991).

De aquí que los objetivos de las compañías se centren en la adquisición, organización y gestión de los recursos en los sectores en los cuales se desenvuelven. Sin embargo, la administración de los recursos empresariales se encuentra relacionada con el término de capacidad. Al respecto, Hafeez, Zhang y Malak (2002) exponen que las capacidades organizacionales se crean mediante el enlace de actividades y procesos. Además, corresponden a las rutinas que las compañías realizan en sus actividades diarias, como se muestra en Nelson y Winter (1982). Según Kaleka (2012), las capacidades corresponden a aquellas actividades que convierten los recursos en acciones estratégicas. No obstante, la interiorización de estas actividades necesitan de un proceso de repetición o rutinas.

Para Nelson y Winter (1982), las rutinas organizacionales son heredables y seleccionables. También representan soluciones exitosas para problemas, como proponen Hafeez, Zhang y Malak (2002). Asimismo, para Teece, Pisano y Shuen (1997) las rutinas se expresan en la posición que tiene la firma en el mercado. Sin ellas los recursos no pueden ser convertidos en innovaciones tecnológicas, como lo proponen Wagner y Weitzel (2007). Al respecto Becker y Zirpoli (2008) argumentan que estas facilitan el proceso de interiorización por parte de los individuos.

En cierto modo, las capacidades no son estáticas y requieren ser gestionadas de manera exclusiva e independiente de los recursos, como lo mencionan Hafeez, Zhang y Malak (2002). Teece, Pisano y Shuen (1997) proponen que las capacidades dinámicas permiten renovar las competencias organizacionales en congruencia con los cambios del ambiente. Estas se establecen para una estrategia y proceso 
específico, como lo proponen Eisenhardt y Martin (2000). Al respecto Winter (2003) manifiesta que el aprendizaje de capacidades puede convertirse en una manera para solucionar los problemas de las compañías.

\subsection{Capacidades de innovación tecnológica}

Para Yam y otros (2004), las organizaciones deben adaptarse e innovar. Para tal fin, se apalancan en las capacidades de innovación tecnológica, las cuales se encuentran conformadas por un conjunto de recursos que incluyen tecnología, productos, procesos, conocimiento y experiencia, lo cual es exhibido por Guan y Ma (2003). Además, para Yam y otros (2010) y Burgelman, Christensen y Wheelwright (2008), este tipo de capacidades corresponden a las particularidades de una organización encargadas de generar innovaciones tecnológicas y generar valor económico para la compañía. Suelen agruparse en bloques que contribuyen con su identificación, uso y entendimiento.

Al respecto Guan y Ma (2003), Yam y otros (2004), y Aguirre y Robledo (2010), proponen que el bloque se encuentra constituido por las capacidades de aprendizaje, I+D, gestión de recursos, manufactura, marketing, organizacional y direccionamiento estratégico. Dicho bloque, será empleado en el desarrollo metodológico que se muestra a continuación

\section{METODOLOGÍA}

La información primaria se obtuvo a partir de diálogos y reuniones, con integrantes de las organizaciones promotoras del Clúster Textil/Confección, Diseño y Moda, organizaciones de apoyo y miembros de Instituciones Universitarias (ver anexo 1). Con la información recabada se procedió con el reconocimiento de las variables de interés; las cuales permitieron desarrollar un modelo de simulación mediante dinámica de sistemas, metodología que fue desarrollada por Forrester (1965). Con todo esto, se procedió con el análisis de escenarios.

\subsection{Hipótesis dinámica}

Para justificar las conexiones causa-efecto, se hace uso de los diagramas causales como lo menciona Sterman (2004). Para el caso específico de las organizaciones pertenecientes al sector textil de la ciudad de Medellín, dichas conexiones se establecieron mediante el estudio de bibliografía especializada y los aportes brindados por expertos del sector Marulanda (2015). (ver tabla 1). 
Modelo para gestionar el conocimiento en el sector textil de Medellín, empleando dinámica de sistemas

Tabla 1. Relaciones causales gestión de conocimiento y capacidades de innovación tecnológica

\begin{tabular}{|c|c|c|}
\hline Conexiones & Fuente & Observaciones \\
\hline 1 & $\begin{array}{l}\text { Yam y otros (2004), Guan y Ma (2003), } \\
\text { Chen, Chen y Lee (2008), Ahmed, } \\
\text { Kristal y Pagell (2014)Wu (2013), } \\
\text { Prašnikar y otros (2008) }\end{array}$ & $\begin{array}{l}\text { - Promocionar y vender productos basado } \\
\text { en la demanda. } \\
\text { - Publicitar, vender productos y lograr la } \\
\text { aprobación de las innovaciones. } \\
\text { - Introducir y ofertar bienes con base en la } \\
\text { comprensión de los requerimientos del } \\
\text { consumidor. } \\
\text { - Comprender las nuevas exigencias de los } \\
\text { consumidores a fin de desarrollar bienes y } \\
\text { procesos con base en la combinación de } \\
\text { recursos. }\end{array}$ \\
\hline 2 & $\begin{array}{l}\text { Terjesen, Patel y Covin (2011), Chen, } \\
\text { Chen y Lee (2008) }\end{array}$ & $\begin{array}{l}\text { - Obtener los objetivos de calidad, costos, } \\
\text { tiempo, volumen y rendimiento. } \\
\text { - Crear metodologías alternativas en los } \\
\text { procesos productivos para optimizar la } \\
\text { calidad. } \\
\text { - Con un mejor desempeño, sobresale la } \\
\text { calidad y crecen las ventas. }\end{array}$ \\
\hline 3 & Yang (2005) & $\begin{array}{l}\text { - Para el desarrollo de las capacidades de } \\
\text { marketing y producción se debe medir la } \\
\text { competencia en el mercado de los nuevos } \\
\text { bienes producidos. } \\
\text { - La medición del comportamiento de los } \\
\text { bienes en el mercado, permite a la empresa } \\
\text { complementar el conocimiento del entorno } \\
\text { con sus conocimientos especializados. }\end{array}$ \\
\hline 4 & von Krogh y Geilinger (2014) & $\begin{array}{l}\text { - Rapidez en la toma de decisiones. } \\
\text { - Reducir el coste de perfeccionamiento de } \\
\text { bienes. } \\
\text { - La combinación de conocimiento explícito } \\
\text { reduce los reprocesos en el área manufac- } \\
\text { tura. }\end{array}$ \\
\hline 5 & $\begin{array}{l}\text { Yam y otros (2004), Bojica y Fuentes } \\
\text { (2012) }\end{array}$ & $\begin{array}{l}\text { - Generar tácticas de I+D y de egresos } \\
\text { asociados con su proceso de perfeccio- } \\
\text { namiento. } \\
\text { - Reconocer las expectativas de los consu- } \\
\text { midores con base a los nuevos desarrollos } \\
\text { tecnológicos. }\end{array}$ \\
\hline
\end{tabular}




\begin{tabular}{|c|c|c|}
\hline Conexiones & Fuente & Observaciones \\
\hline 6 & $\begin{array}{l}\text { Ooi (2014), Maurer (2010), Guan y } \\
\text { Ma (2003), Guan y otros (2006), Ford, } \\
\text { Wells y Capper (2003) }\end{array}$ & $\begin{array}{l}\text { - Generar bienes y nuevas alianzas estraté } \\
\text { gicas. } \\
\text { - Obtener conocimiento del personal, con- } \\
\text { sumidores y proveedores para perfeccionar } \\
\text { la calidad de los productos y servicios. } \\
\text { - Incorporar metodologías para afrontar las } \\
\text { variaciones del ambiente mercantil. }\end{array}$ \\
\hline 7 & $\begin{array}{l}\text { Yam y otros (2004), Kocoglu y otros } \\
\text { (2012), Lukas y Bell (2000) }\end{array}$ & $\begin{array}{l}\text { - Generar tácticas de I+D y de egresos } \\
\text { asociados con su proceso de perfeccio- } \\
\text { namiento. } \\
\text { - Exhibir nuevos bienes y servicios, o difun- } \\
\text { dir los ya existentes. } \\
\text { - Invertir en I+D y acumular Nuevo conoci- } \\
\text { miento tecnológico. }\end{array}$ \\
\hline 8 & Yan-Rong y Qiao-Ling (2009) & $\begin{array}{l}\text { Reducir el riesgo relacionado con las de- } \\
\text { cisiones de innovación, combinar diversas } \\
\text { clases de conocimiento y mejorar las } \\
\text { habilidades individuales. }\end{array}$ \\
\hline 9 & Guan y Ma (2003) & $\begin{array}{l}\text { - Incorporar metodologías para afrontar las } \\
\text { variaciones del ambiente mercantil. }\end{array}$ \\
\hline 10 & $\begin{array}{l}\text { Yakhlef (2007), Mahesh y Suresh, } \\
\text { (2004), Nwankpa y Roumani (2014), } \\
\text { Blome, Schoenherr y Eckstein (2014) }\end{array}$ & $\begin{array}{l}\text { - Generar mecanismos de coordinación } \\
\text { y comunicación entre el personal de las } \\
\text { empresas. } \\
\text { - Generar estructuras apropiadas de comu- } \\
\text { nicación para simplificar la transferencia } \\
\text { de conocimiento. }\end{array}$ \\
\hline $\begin{array}{c}\text { Demás } \\
\text { conexiones }\end{array}$ & $\begin{array}{l}\text { Consulta a expertos sector textil e } \\
\text { innovación. }\end{array}$ & \\
\hline
\end{tabular}

Fuente. elaboración propia

Con todo esto, las relaciones causales del modelo propuesto de gestión de conocimiento y las capacidades de innovación tecnológica, posee en conjunto ciclos de realimentación positivos o de refuerzo (ver figura 1.). Por ende, que las decisiones que se tomen en el manejo de una variable, repercuten directamente en el crecimiento o decrecimiento proporcional en sus variables asociadas. 
Figura 1. Diagrama causal gestión de conocimiento y capacidades de innovación tecnológica

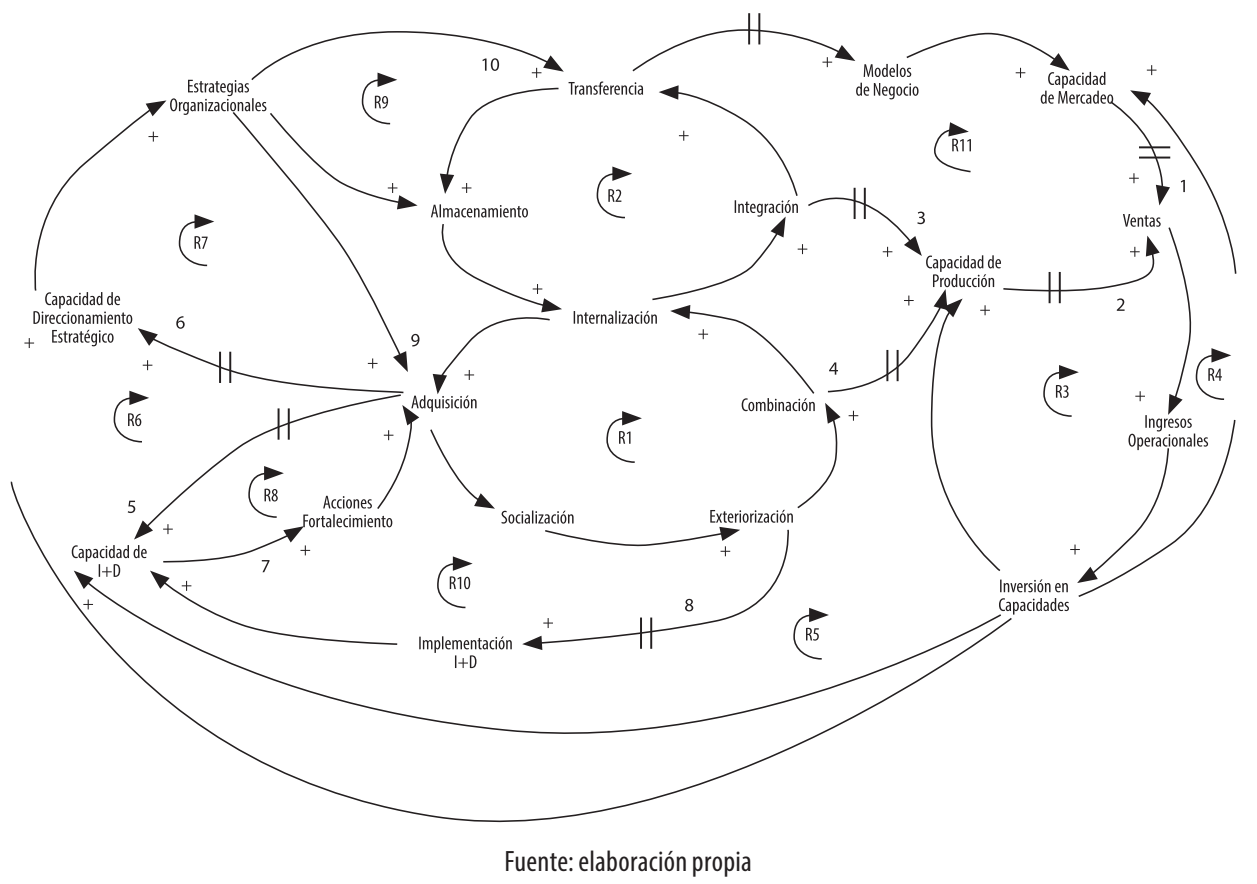

Ahora bien, el diagrama causal exhibe el conocimiento como la base para el mejoramiento de las cuatro capacidades de innovación tecnológica que se emplearon en la investigación. A continuación se empleará la simulación a partir de diagramas de flujos y niveles, desarrollada por Forrester (1965), la cual permitirá identificar el nivel actual del sector con respecto a la coordinación de metodologías de gestión de conocimiento y capacidades de innovación tecnológica. La simulación y los alcances de los escenarios, se exhibirán en las secciones subsiguientes.

\subsection{Diagrama de Forrester}

El diagrama causal expuesto anteriormente brinda una perspectiva extensa del objeto de la investigación. No obstante, se debe acompañar de magnitudes para las variables y factores que relacionan las fases de gestión de conocimiento y las capacidades de innovación tecnológica' Para tal fin, se empleará el Software de Simulación PowerSim Studio $10^{\circledR}$.

1 Los datos e información empleados en el desarrollo de las simulaciones, se suministrarán a los interesados bajo autorización expresa de los autores, a través del correo electrónico: nmarulandagr@unal. edu.co. 
Se usó un horizonte temporal y tiempo de paso de uno y diez años. Se estableció un conjunto de cinco variables de interés: estrategias organizacionales, canales de comunicación, formación, cultura y acciones de fortalecimiento Marulanda (2015). Los valores de las variables de interés se hallaron mediante la normalización de unidades con un valor máximo de 1 y un nivel de cumplimiento de 5 .

Debido al tamaño del diagrama de Forrester, el modelo se separó en dos diagramas: Fases de gestión de conocimiento y fases-capacidades de innovación. En el caso específico de la fase de adquisición de conocimiento (ver figura 2), se observa que los valores se restringen por el parámetro de adquisición máxima. Asimismo, el parámetro de pérdida de adquisición el grado al cual el sector textil deja de adquirir conocimiento. Por su parte, para la capacidad de producción (ver figura 3), se muestra que su desempeño se encuentra apalancado por el porcentaje de inversión que se destina para su desarrollo, y por el efecto acumulado de las fases de gestión de conocimiento en dicha capacidad.

Figura 2. Diagrama de Forrester para adquisición de conocimiento

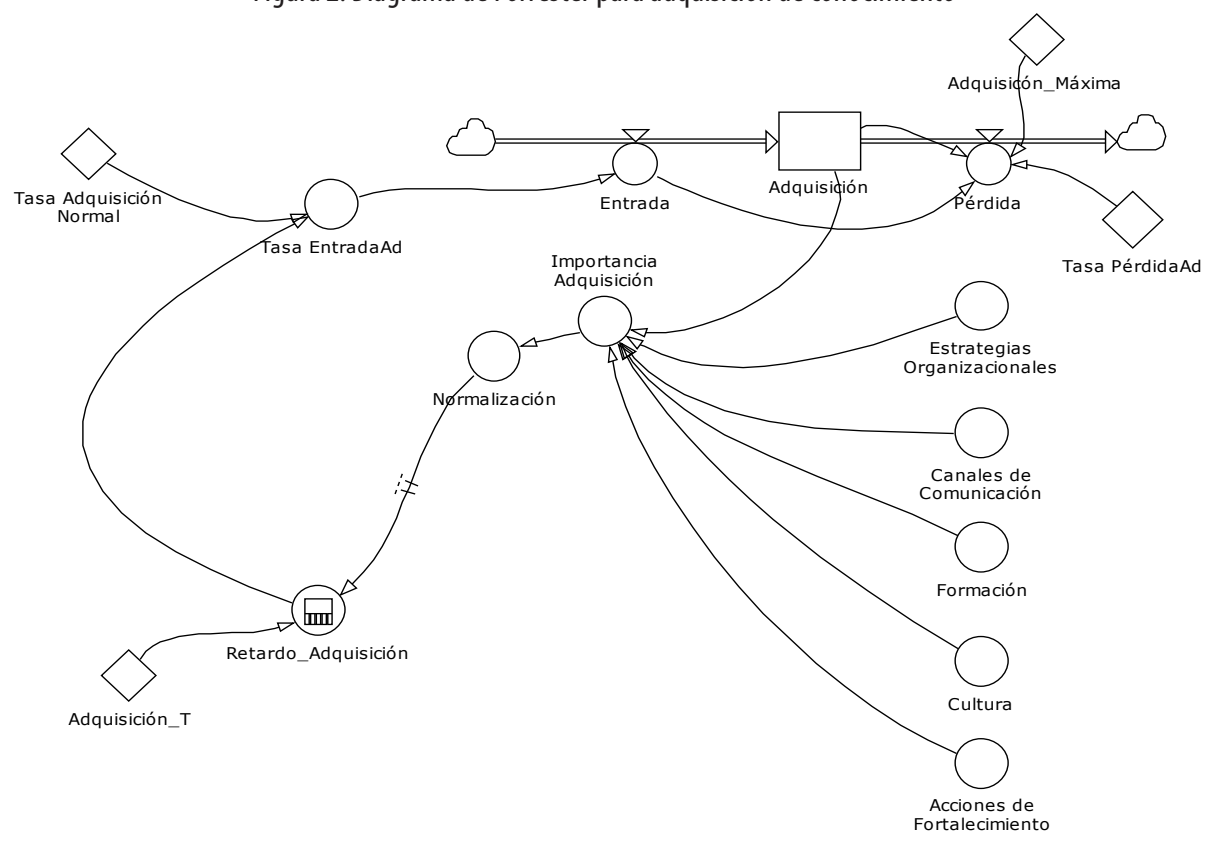

Fuente: elaboración propia 
Figura 3. Diagrama de Forrester para capacidad de producción

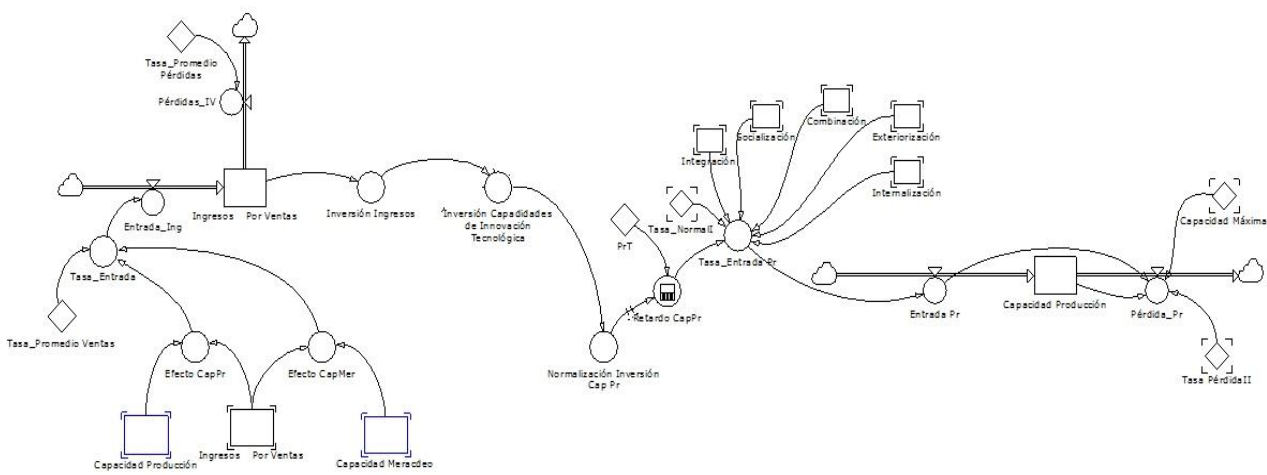

Fuente: elaboración propia

\subsection{Validación del modelo}

Se realizó la validación de modelo mediante pruebas de consistencia dimensional donde se evidenció que todas las variables, ecuaciones, flujos y niveles, contaban con las unidades indicadas. Asimismo, mediante las pruebas de condiciones extremas, el modelo permaneció estable y los resultados se ajustaban al sistema real. Con todo esto, en la consulta realizada a expertos sobre el comportamiento, ellos manifestaron que los resultados se comportan de manera similar a las condiciones actuales del sector, en el cual en términos generales no se implementan estrategias para gestionar el conocimiento, o se realiza de forma empírica.

\section{RESULTADOS}

\subsection{Análisis de escenarios}

Con base en los supuestos del modelo, el diagrama causal y el diagrama de Forrester, en esta sección se abordaran diversos escenarios para identificar las conductas de las organizaciones del sector textil en la ciudad de Medellín en lo que se refiere al uso de estrategias de gestión de conocimiento en la acumulación de capacidades de innovación tecnológica, y el papel que desempeñan en los ingresos operacionales totales del sector.

\subsubsection{Escenario Base}

El horizonte temporal establecido de diez años, no es idóneo en la obtención de los objetivos de acumulación que debe ser equivalente a 5 (ver figura 4). El anterior contexto, es acorde a las opiniones expuestas por el grupo de expertos, pues en el sector se presenta un desconocimiento general sobre el término de gestión de 
conocimiento. El uso de esta metodología se encuentra sujeto a varios elementos, entre estos están el aumento en los valores de los niveles de las variables comunes: estrategias organizacionales, canales de comunicación, formación, cultura y acciones de fortalecimiento en I+D, Marulanda (2015).

Figura 4. Acumulación de conocimiento escenario base

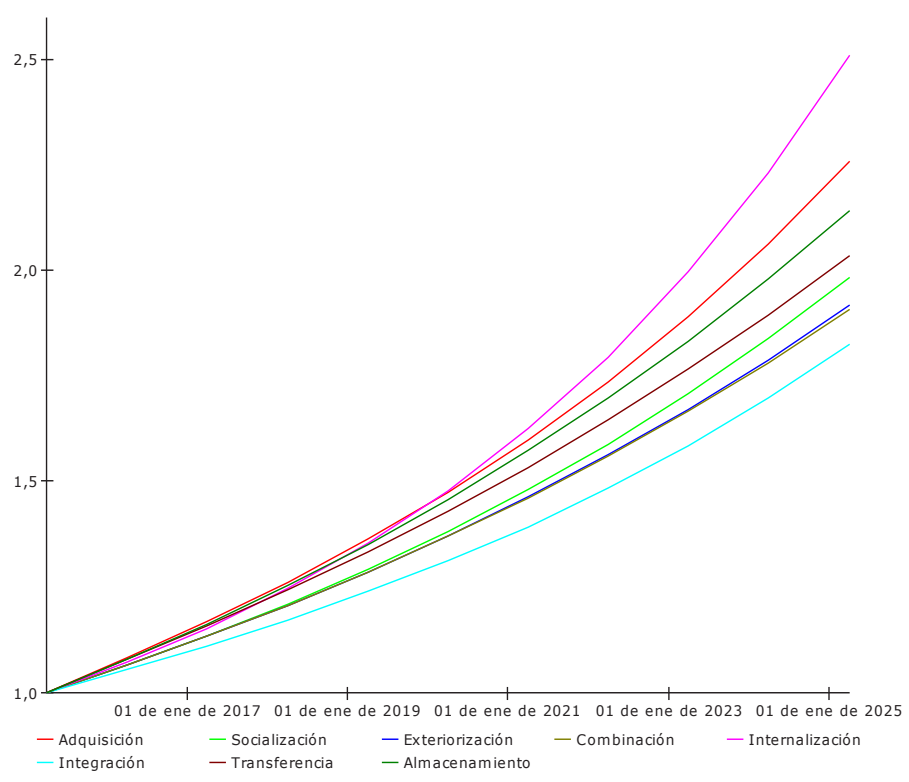

Fuente: elaboración propia

Paralelamente, la acumulación de capacidades de innovación tecnológica se acercó al valor esperado de 5, en el horizonte temporal de simulación (ver figura 5). Lo anterior se da porque en términos generales, las organizaciones del sector textil han comenzado a crear e implementar métodos de innovación más complejos, que no solo abarcan el diseño y confección de vestuario. Un caso especial, es el desarrollo de telas inteligentes y bienes que reducen los impactos sobre el medio ambiente. 
Figura 5. Acumulación de capacidades de innovación tecnológica escenario base

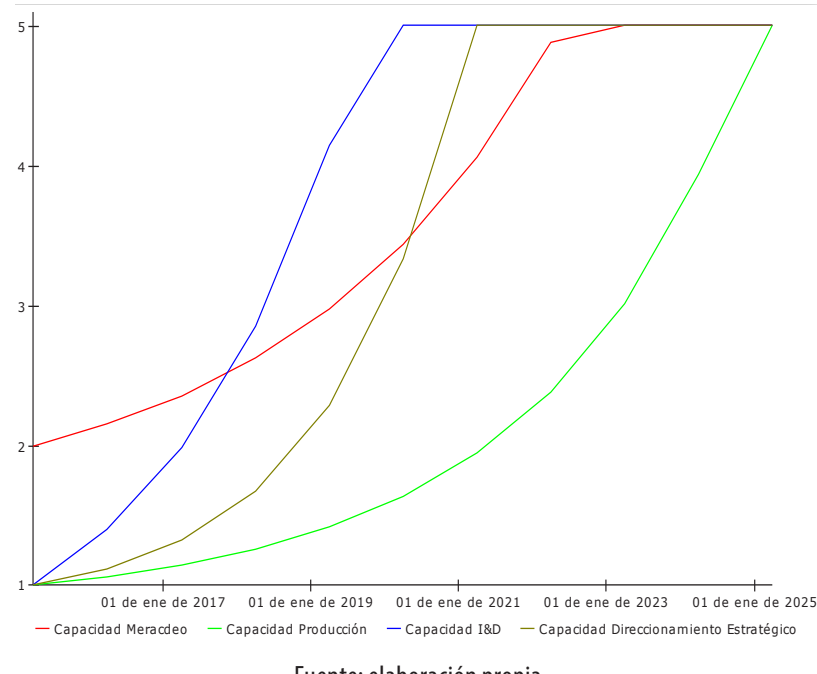

Fuente: elaboración propia

\subsubsection{Escenario 1}

Mediante este escenario se espera identificar si existe algún afecto en el sistema, al aumentar el valor de las variables comunes de al nivel máximo y el porcentaje de inversión en capacidades de innovación tecnológica (ver tabla 2).

Tabla 2. Valores iníciales variables de interés

\begin{tabular}{|c|c|c|}
\hline Variable & Valor normalizado & Valor esperado \\
\hline Estrategias Organizacionales & 2 & 5 \\
\hline Canales de Comunicación & 2 & 5 \\
\hline Formación & 2 & 5 \\
\hline Cultura & 4 & 5 \\
\hline Acciones de Fortalecimiento en I+D & 1 & 5 \\
\hline \% Inversión Cap. Innovación Tec. & $2,68 \%$ & $7 \%$ \\
\hline
\end{tabular}

Fuente. Elaboración propia

Entre los resultados al adoptar las estrategias de este escenario se encuentra una aproximación al nivel máximo acumulable (5) en términos de conocimiento y se observa su estabilización en el horizonte temporal predeterminado (ver figura 6). El efecto que tiene la implementación de esta alternativa sobre los ingresos totales del sector es sobresaliente, pues estos alcanzaron un monto de $2 \times 10^{21} \mathrm{COP}$ \$ (ver figura 7). 
Figura 6. Acumulación de conocimiento escenario 1

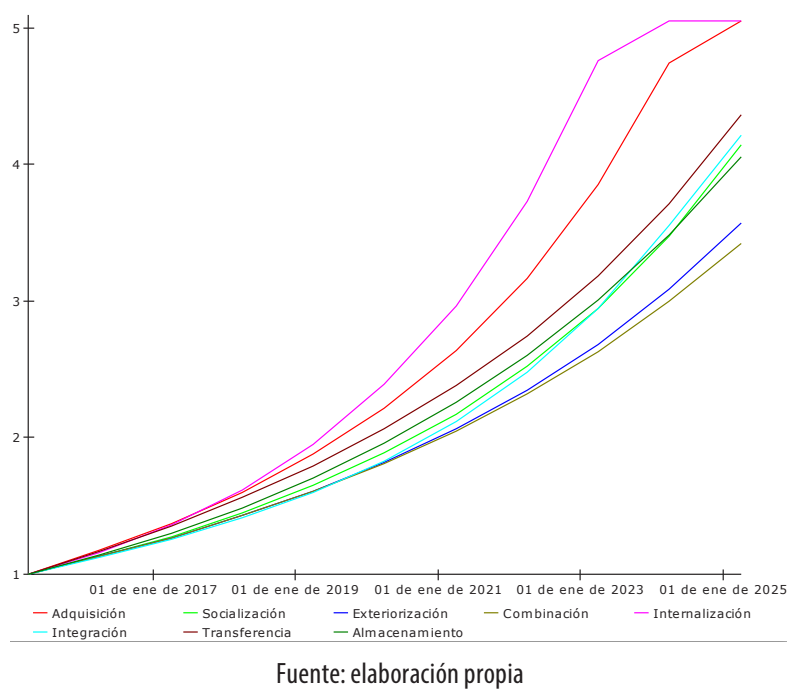

Figura 7. Ingresos operacionales escenario 1

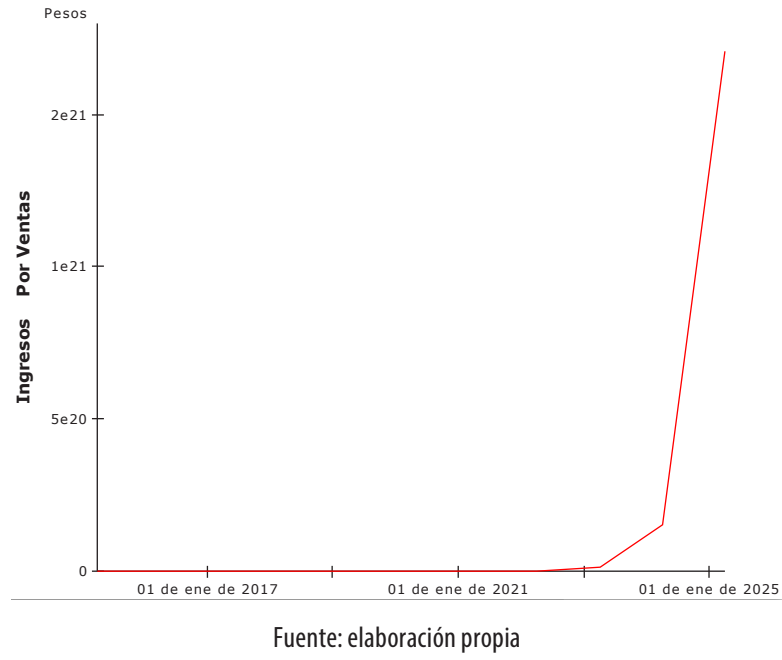

La adopción de esta alternativa favorece al sector textil, pues activa el proceso de acumulación de capacidades de innovación tecnológica, y les ayuda a obtener su nivel máximo en un horizonte temporal inferior al previamente establecido (Ver Figura 8.). 
Figura 8. Acumulación de capacidades de innovación tecnológica escenario 1

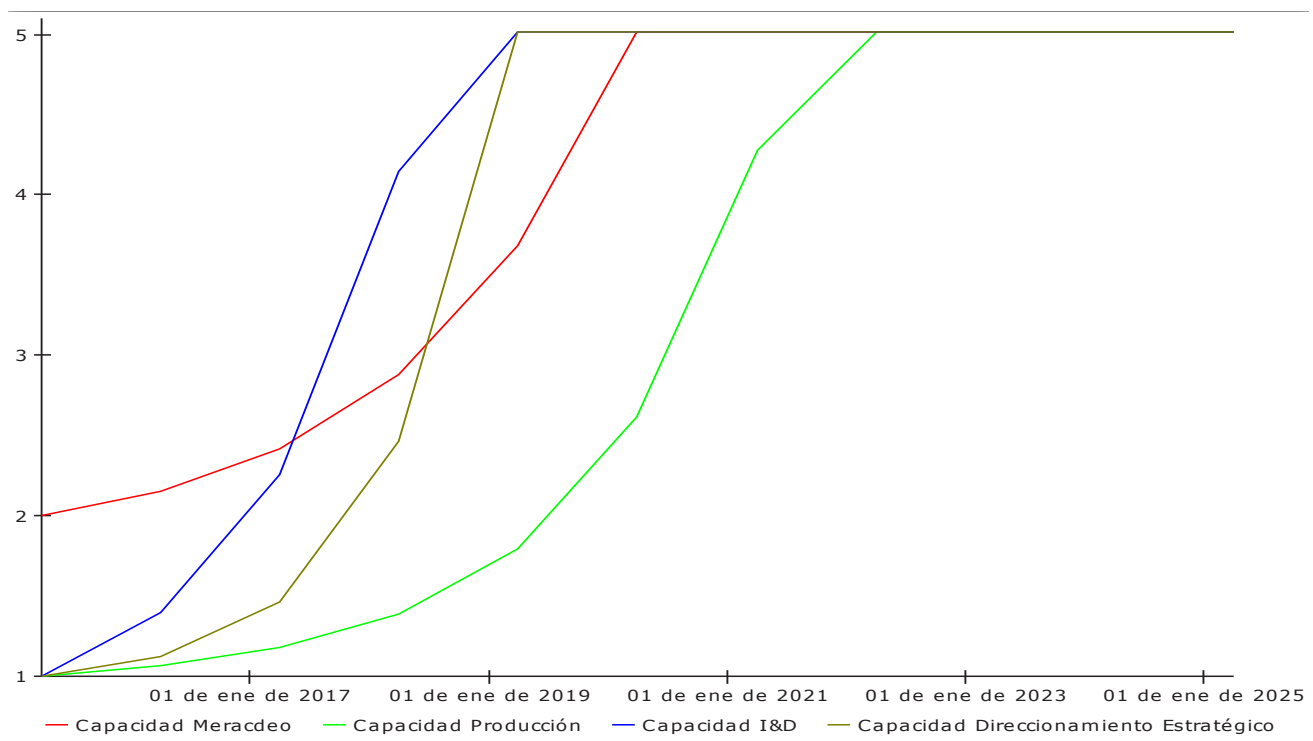

Fuente: elaboración propia

\subsubsection{Escenario 2}

Las variables comunes de interés poseen diversos montos de importancia en cada una de las fases de gestión de conocimiento (ver tabla 3). Mediante el desarrollo de este escenario se desea analizar si la estandarización del nivel de importancia a 0,8, tiene algún efecto en la acumulación de conocimiento, capacidades de innovación tecnológica y nivel de ingresos por ventas.

Tabla 3. Niveles de importancia variables comunes de interés

\begin{tabular}{|c|c|c|c|c|c|c|c|c|}
\hline Variables & $\begin{array}{c}\text { Importancia } \\
\text { adquisición }\end{array}$ & $\begin{array}{c}\text { Importancia } \\
\text { socialización }\end{array}$ & $\begin{array}{c}\text { Importancia } \\
\text { exterioriza- } \\
\text { ción }\end{array}$ & $\begin{array}{c}\text { Importancia } \\
\text { combinación }\end{array}$ & $\begin{array}{c}\text { Importancia } \\
\text { integración }\end{array}$ & $\begin{array}{c}\text { Importancia } \\
\text { integración }\end{array}$ & $\begin{array}{c}\text { Impoetancia } \\
\text { transferencia }\end{array}$ & $\begin{array}{c}\text { Importancia } \\
\text { almacena- } \\
\text { miento }\end{array}$ \\
\hline $\begin{array}{c}\text { Estrategias } \\
\text { organiza- } \\
\text { cionales }\end{array}$ & 0,94 & 0,11 & 0,11 & 0,11 & 0,11 & 0,14 & 0,38 & 0,14 \\
\hline $\begin{array}{c}\text { Canales de } \\
\text { comunica- } \\
\text { ción }\end{array}$ & 0,35 & 0,77 & 0,77 & 0,77 & 0,77 & 0,64 & 0,95 & 0,95 \\
\hline Formación & 0,19 & 0,91 & 0,91 & 0,91 & 0,91 & 0,2 & 0,47 & 0,2 \\
\hline
\end{tabular}


Natalia Marulanda Grisales - Iván A. Montoya Restrepo

\begin{tabular}{|c|c|c|c|c|c|c|c|c|}
\hline Variables & $\begin{array}{c}\text { Importancia } \\
\text { adquisición }\end{array}$ & $\begin{array}{c}\text { Importancia } \\
\text { socialización }\end{array}$ & $\begin{array}{c}\text { Importancia } \\
\text { exterioriza- } \\
\text { ción }\end{array}$ & $\begin{array}{c}\text { Importancia } \\
\text { combinación }\end{array}$ & $\begin{array}{c}\text { Importancia } \\
\text { integración }\end{array}$ & $\begin{array}{c}\text { Importancia } \\
\text { integración }\end{array}$ & $\begin{array}{c}\text { Impoetancia } \\
\text { transferencia }\end{array}$ & $\begin{array}{c}\text { Importancia } \\
\text { almacena- } \\
\text { miento }\end{array}$ \\
\hline Cultura & 0,8 & 0,45 & 0,45 & 0,45 & 0,45 & 0,4 & 0,58 & 0,98 \\
\hline $\begin{array}{c}\text { Acciones } \\
\text { de forta- } \\
\text { lecimiento } \\
\text { i+d }\end{array}$ & 0,92 & 0,06 & 0,68 & 0,68 & 0,68 & 0,74 & 0,73 & 0,29 \\
\hline
\end{tabular}

Fuente: elaboración propia.

El presente escenario representa un mejor comportamiento de los resultados al tener como referente al escenario base (ver figura 9). La acumulación de conocimiento mejora con respecto al valor objetivo. También presentaron mejoras los niveles de capacidades de innovación tecnológica. Por ejemplo, la capacidad de producción logra el nivel máximo posible y se estabiliza un año antes del cumplimiento del horizonte temporal de simulación, el año 2024 (ver figura 10).

Figura 9. Acumulación de conocimiento escenario 2

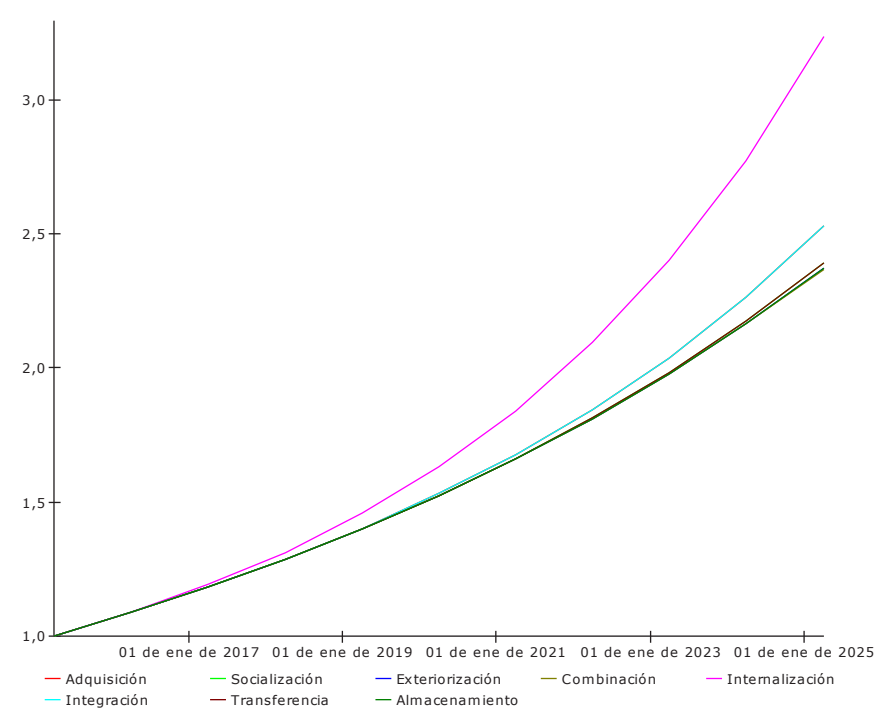

Fuente: elaboración propia 
Figura 10. Acumulación de capacidades de innovación tecnológica escenario 2

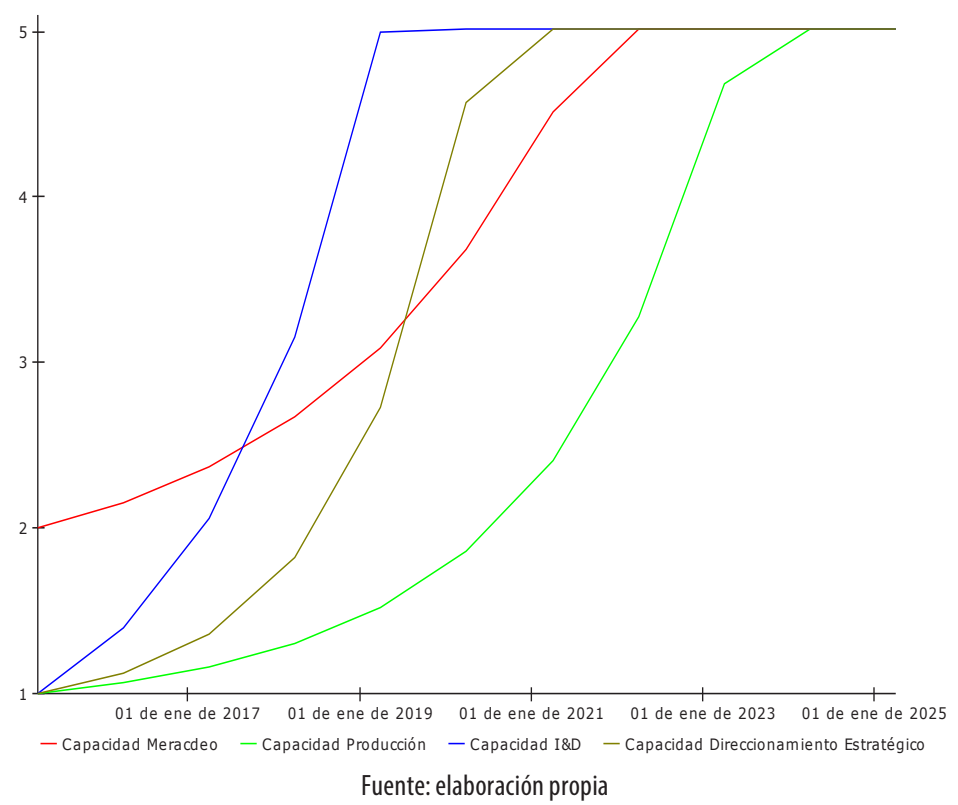

Esta alternativa también generó incrementos en los ingresos operacionales del sector textil, al compararse con los resultados del escenario base, pues estos alcanzaron un monto de $6 \times 10^{20} \mathrm{COP} \$$ (Ver Figura 11.). Aunque es una buena suma, el presente escenario no igualó el buen desempeño del nivel ingresos operacionales bajo el escenario 1.

Figura 11. Ingresos operacionales por ventas escenario 2

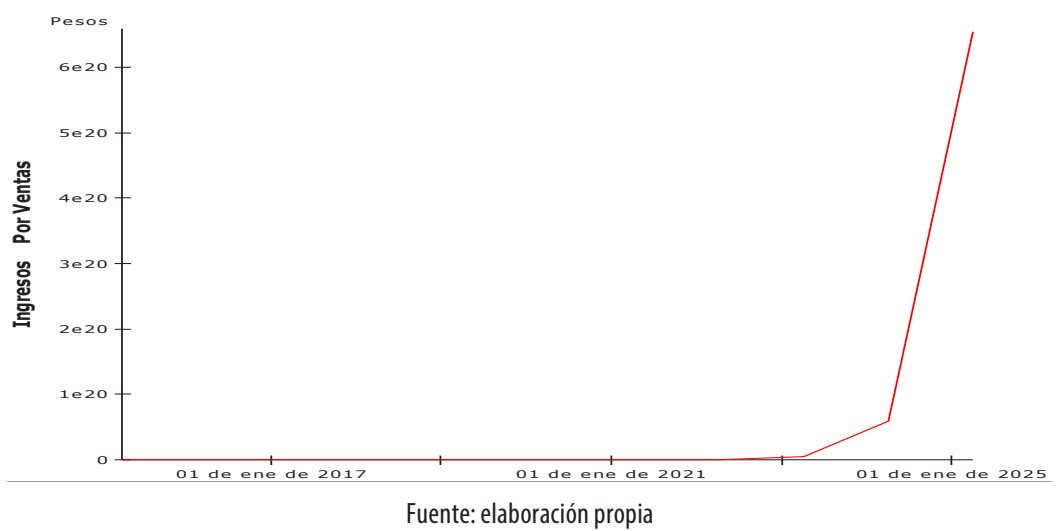




\subsubsection{Escenario 3}

Con base en los resultados del escenario 2, se asignará un valor estándar de 0,8 a los niveles de importancia. Todas las variables comunes de interés adquirirán un monto de 4 (ver tabla 4).

Tabla 14. Valores variables de interés escenario 3

\begin{tabular}{|l|c|c|}
\hline \multicolumn{1}{|c|}{ Variable } & Valor normalizado & Valor deseado \\
\hline Estrategias Organizacionales & 2 & 4 \\
\hline Canales de Comunicación & 2 & 4 \\
\hline Formación & 2 & 4 \\
\hline Cultura & 4 & 4 \\
\hline Acciones de Fortalecimiento en I+D & 1 & 4 \\
\hline
\end{tabular}

Fuente. Elaboración propia

Con esta alternativa de simulación, se obtuvieron incrementos relevantes en la acumulación de conocimiento en las fases estudiadas, pues algunas de ellas lograron el monto máximo posible en el periodo de simulación establecido (ver figura 12). Además mejoró la acumulación de capacidades de innovación tecnológica, al alcanzar los objetivos en periodos de tiempo inferiores a los referenciados en el escenario base (ver figura 13)

Figura 12. Acumulación de conocimiento escenario 3

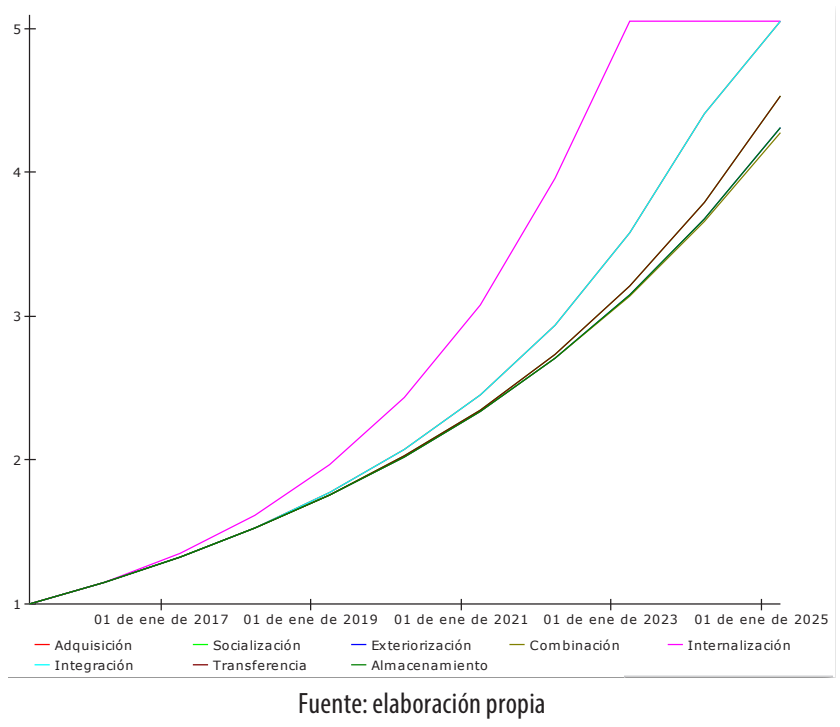


Figura 13. Acumulación de capacidades de innovación tecnológica escenario 3

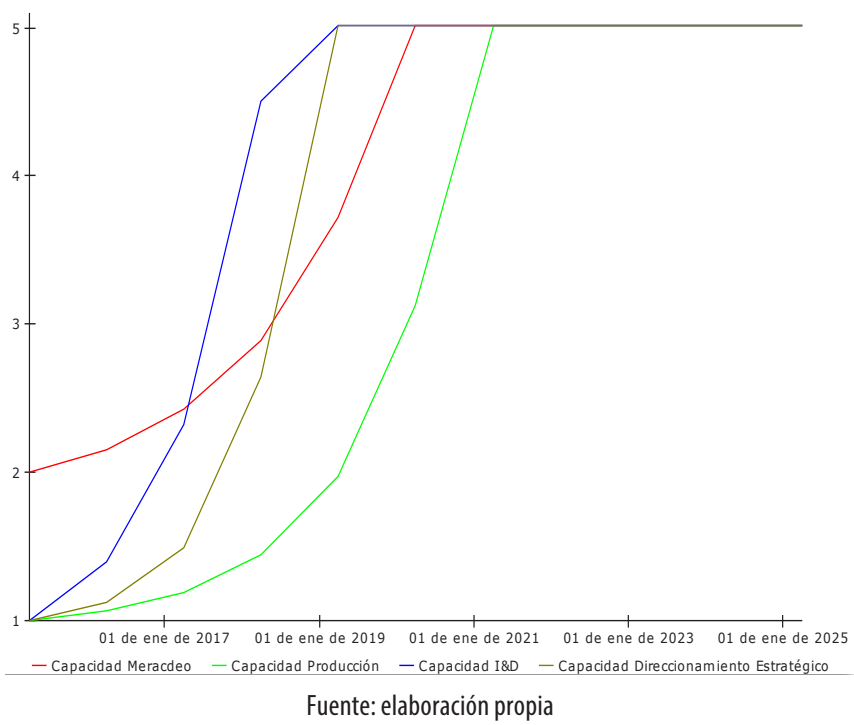

Para finalizar, los ingresos totales por ventas en el sector fueron equivalentes a los obtenidos en el escenario 1, es decir un monto de $2 \times 10^{21} \mathrm{COP} \$$ (ver figura 14).

Figura 14. Ingresos operacionales por ventas escenario 3

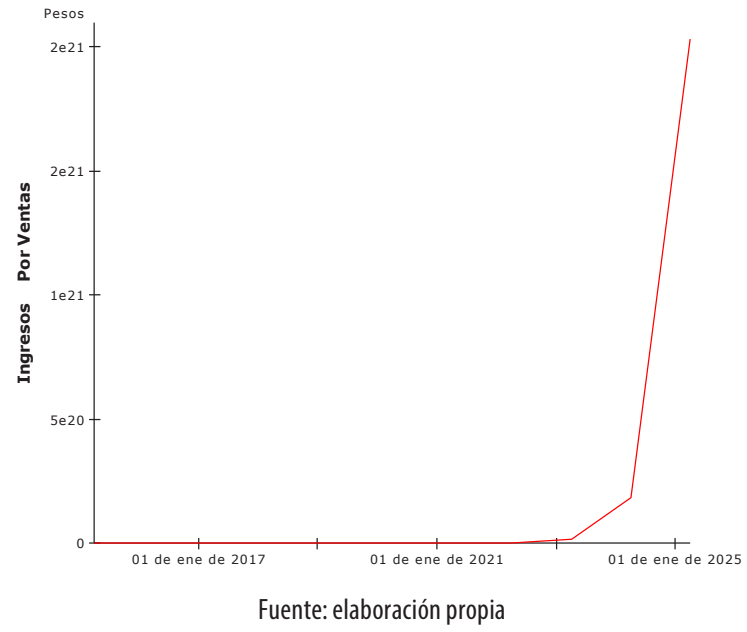




\section{CONCLUSIONES}

Con el uso de dinámica de sistemas como herramienta cualitativa y cuantitativa de investigación, se logró demostrar la una relación de causalidad positiva entre las fases de gestión de conocimiento y las capacidades de innovación tecnológica, lo cual permitió mejorar el desempeño de las empresas objeto de estudio con respecto a los ingresos operacionales. Lo anterior se deriva de los diferentes modelos de simulación y conjuntos de escenarios que fueron empleados.

Los alcances de las alternativas establecidas en la simulación de escenarios, contribuyeron con la determinación de aquellas características que facilitan la acumulación de conocimiento y capacidades de innovación tecnológica en las organizaciones del sector textil en la ciudad de Medellín. No obstante, se debe aclarar que aunque las conclusiones obtenidas a partir del proceso de simulación son beneficiosas para las compañías, estas provienen de escenarios optimistas que actualmente no se adaptan a las condiciones de las empresas del sector, con respecto al tratamiento que les han dado a las variables de interés comunes (Estrategias organizacionales, canales de comunicación, formación, cultura y acciones de fortalecimiento en I+D).

Aunque son varios los modelos que se han propuesto para gestionar el conocimiento, estos han resultado ser de difícil aplicación en algunos sectores empresariales debido a las particularidades inherentes a cada compañía. Sin embargo, es posible abstraer las mejores características de cada uno de los modelos existentes y adaptarlas a las condiciones propias de cada organización, como ocurrió en la ejecución de los modelos causales y de simulación de la presente investigación.

Si bien el modelo de simulación empleado fue desarrollado con base en las condiciones actuales del sector textil en la ciudad de Medellín, este puede ser replicado en cualquier otro sector u organización debido a las características generales que presenta en su concepción y formulación.

\section{BIBLIOGRAFÍA}

Aguirre, J. J. y Robledo, J. (2010). Metodología para medir y evaluar las capacidades tecnológicas de innovación aplicando sistemas de lógica difusa caso fábricas de software. Universidad Nacional de Colombia, 90 p.

Ahmed, M. U; Kristal, M. M. y Pagell, M. (2014). Impact of operational and marketing capabilities on firm performance: Evidence from economic growth and downturns. En: International Journal of Production Economics, Vol.154, p. 59-71.

Andreeva, T., y Ikhilchik, I. (2011). Applicability of the SECI Model of knowledge creation in Russian cultural context: Theoretical analysis. En:Knowledge and Process Management, Vol.18, No.1, p. 56-66. 
Modelo para gestionar el conocimiento en el sector textil de Medellín, empleando dinámica de sistemas

Arnett, D. B., y Wittmann, C. M. (2014). Improving marketing success: The role of tacit knowledge exchange between sales and marketing. En: Journal of Business Research, Vol. 67, No.3, p. 324-331.

Barney, J. (1991). Firm Resources and Sustained Competitive Advantage. En: Journal of Management, Vol.17, No.1, p. 99-120.

Becker, M. C. y Zirpoli, F. (2008). Applying organizational routines in analyzing the behavior of organizations. En: Journal of Economic Behavior \& Organization, Vol. 66, No.1, p. 128-148.

Betancur, J. y Vargas, I. (2012). Gestión tecnológica en el sector textil de Medellín (Colombia). La visión de los empresarios. En: Revista de la Escuela Colombiana de Ingeniería, p.85, 58.

Blome, C., Schoenherr, T., y Eckstein, D. (2014). The impact of knowledge transfer and complexity on supply chain flexibility: A knowledge-based view. En: International Journal of Production Economics, Vol.147, Part B, p. 307-316.

Bojica, A. M., y Fuentes, M. del M. F. (2012). Knowledge acquisition and corporate entrepreneurship: Insights from Spanish SMEs in the ICT sector. En: Journal of World Business, Vol.47, No. 3, p. 397-408.

Burgelman, R. A., Christensen, C. M., y Wheelwright, S. C. (2008). Strategic Management of Technology and Innovation (Edición: 5). Boston: McGraw-Hill Higher Education, p.1280.

Checkland, P. (1981). Systems thinking, systems practice. J. Wiley, p. 424.

Chen, L.-J., Chen, C.-C., y Lee, W.-R. (2008). Strategic Capabilities, Innovation Intensity, and Performance of Service Firms. En: Journal of Service Science and Management, Vol.1, No.2, p. $111-122$.

Delen, D., Zaim, H., Kuzey, C., y Zaim, S. (2013). A comparative analysis of machine learning systems for measuring the impact of knowledge management practices. En: Decision Support Systems, Vol. 54, No.2, p. 1150-1160.

Drucker, P. F. (1988). The coming of the new organization. En: Harvard Business Review.

Eisenhardt, K. M., y Martin, J. A. (2000). Dynamic capabilities: what are they? En: Strategic Management Journal, Vol. 21, No. 10, p. 1105-1121.

Fleck, J. (1996). Informal information flow and the nature of expertise in financial services. En: International Journal of Technology Management, Vol.11, No.1, p. 104-128.

Ford, E. W., Wells, R., y Capper, S. A. (2003). High performance public health: Assessing agencies' strategic management capabilities. En: Journal of Health and Human Services Administration, Vol.25, No.4, p. 407-431.

Forrester, W. J. (1965). Industrial Dynamics. M.I.T. Press, Mass institute of technology.

Gourlay, S. (2003). The SECI model of knowledge creation: some empirical shortcomings. En: 4th European Conference on knowledge management.

Gourlay, S. (2006). Towards conceptual clarity concerning tacit knowledge: a review of empirical studies. En: Knowledge Management Research E Practice, Vol.4, No.1, p. 60-69. 
Grant, R. M. (1991). Resource-Based Theory of Competitive Advantage: Implications for Strategy Formulation. En: Harvard Business Review.

Grant, R. M. (2002). Contemporary Strategy Analysis. Hoboken, N.J.; Chichester: Wiley, p. 516.

Guan, J. C., Yam, R. C. M., Mok, C. K., y Ma, N. (2006). A study of the relationship between competitiveness and technological innovation capability based on DEA models. En: European Journal of Operational Research, Vol.170, No. 3, p. 971-986.

Guan, J., y Ma, N. (2003). Innovative capability and export performance of Chinese firms. En: Technovation, Vol. 23, No.9, p. 737-747.

Hafeez, K., Zhang, Y., y Malak, N. (2002). Determining key capabilities of a firm using analytic hierarchy process. En: International Journal of Production Economics, Vol.76, No.1, p. 39-51.

Hedlund, G. (1994). A model of knowledge management and the N-form corporation. En: Strategic Management Journal, Vol.15, No.2, p. 73-90.

Howells, J. (1996). Tacit knowledge, innovation and technology transfer. En: Technology Analysis E Strategic Management, Vol.8, No.2, p. 91-106.

INEXMODA. (2011). Caracterización Económica de la Cadena productiva hacia un sistema moda. En: Inexmoda.

INEXMODA. (2011a). El sector textil/confección en Colombia. Retos y oportunidades. En: Ine xmoda

Kaleka, A. (2012). Studying resource and capability effects on export venture performance. En: Journal of World Business, Vol.47, No.1, p. 93-105.

Kocoglu, I., Imamoglu, S. Z., Ince, H., y Keskin, H. (2012). Learning, RED and Manufacturing Capabilities as Determinants of Technological Learning: Enhancing Innovation and Firm Performance. En: Procedia - Social and Behavioral Sciences, Vol.58, p. 842-852.

Lukas, B. A., y Bell, S. J. (2000). Strategic Market Position and RED Capability in Global Manufacturing Industries: Implications for Organizational Learning and Organizational Memory. En: Industrial Marketing Management, Vol.29, No.6, p. 565-574.

Mahesh, K., y Suresh, J. K. (2004). What is the K in KM Technology. En: Electronic Journal of Knowledge Management, Vol.2, p.11-22.

Manzanares, M. J. D., y Gómez, F. G. (2008). Gestión Del Conocimiento Organizativo, Innovación Tecnológica Y Resultados. Una Investigación Empírica. En: Investigaciones Europeas de Dirección y Economía de la Empresa, p. 139-167.

Marino, K. E. (1996). Developing consensus on firm competencies and capabilities. En: The Academy of Management Executive, Vol.10, No.3, p. 40-51.

Martin, L., y Root, D. (2009). Knowledge creation in construction: The SECI model. En: 25th Annual ARCOM conference, p. 7-9.

Marulanda, N. (2015). Modelo de gestión de conocimiento en el sector textil en la ciudad de Medellín empleando dinámica de sistemas. En: Universidad Nacional de Colombia - Sede Medellín. 
Modelo para gestionar el conocimiento en el sector textil de Medellín, empleando dinámica de sistemas

Maurer, I. (2010). How to build trust in inter-organizational projects: The impact of project staffing and project rewards on the formation of trust, knowledge acquisition and product innovation. En: International Journal of Project Management, Vol.28, No.7, p. 629-637.

Mejía, A., Montoya, A., y Bravo, M. (2011). Innovación Tecnológica y Mejoramiento Productivo: una perspectiva para el desarrollo de la competitividad en la pyme. En: Ninth LACCEI Latin American an Caribbean Conference (LACCEI), Engineering for a Smart Planet, Innovation, Information Technology and Computational Tools for Sustainable Development, Medellín, Colombia.

MCIT- Ministerio de Comercio, Industria y Turismo. (2009). Informe final sector textil, confección, diseño y moda. Bogotá Colombia.

Nelson, R. R., y Winter, S. G. (1982). An Evolutionary Theory of Economic Change. Cambridge, Mass.: Belknap Press.

Nonaka, I., y Takeuchi, H. (1995). The Knowledge-Creating Company: How Japanese Companies Create the Dynamics of Innovation. New York: Oxford University Press, p.284.

Nwankpa, J., y Roumani, Y. (2014). Understanding the link between organizational learning capability and ERP system usage: An empirical examination. En: Computers in Human Behavior, Vol.33, p. 224-234.

Ooi, K.-B. (2014). TQM: A facilitator to enhance knowledge management? A structural analysis. En: Expert Systems with Applications, Vol. 41, No.11, p. 5167-5179.

Penrose, E. (1959). The Theory of the Growth of the Firm. Oxford University Press.

Polanyi, M. (1966). The Tacit Dimension. University of Chicago Press.

Prašnikar, J., Lisjak, M., Buhovac, A. R., y Štembergar, M. (2008). Identifying and Exploiting the Inter relationships between Technological and Marketing Capabilities. En: Long Range Planning, Vol.41, No.5, p. 530-554.

Sánchez, P. (2007). Medición y difusión del capital intelectual en las pequeñas y medianas empresas: un camino para incrementar la I+ D Recomendaciones de la Unión Europea. En: Revista Madri+d, No.40.

Shao, Z., Feng, Y., y Liu, L. (2012). The mediating effect of organizational culture and knowledge sharing on transformational leadership and Enterprise Resource Planning systems success: An empirical study in China. En: Computers in Human Behavior, Vol.28, No.6, p. 2400-2413.

Sterman, J. (2004). Business Dynamics: Systems Thinking and Modeling for a Complex World. Boston, McGraw-Hill, p.1008.

Sternberg, R. J. (2000). Practical Intelligence in Everyday Life. Cambridge University Press.

Sundaresan, S., y Zhang, Z. (2012). Parallel teams for knowledge creation: Role of collaboration and incentives. En: Decision Support Systems, Vol.54, No.1, p.109-121.

Teece, D. J., Pisano, G., y Shuen, A. (1997). Dynamic capabilities and strategic management. En: Strategic Management Journal, Vol.18, No.7, p. 509-533. 
Tejedor, B., y Aguirre, A. (1998). Proyecto Logos: investigación relativa a la capacidad de aprender de las empresas españolas. En: Boletín de Estudios Económicos, Vol.53, No.164, p.231-249.

Terjesen, S., Patel, P. C., y Covin, J. G. (2011). Alliance diversity, environmental context and the value of manufacturing capabilities among new high technology ventures. En: Journal of Operations Management, Vol.29, No.1, p.105-115.

Vargas, J. F. (2013). Análisis de oportunidades de mejora para el clúster textil, confección, diseños y moda en Medellín, de acuerdo a las tendencias de la moda en el ámbito local, Medellín. Universidad EAFIT.

Vélez Cabrera, L. G., Rodríguez López, E. F., Camacho Ríos, M. T., y Cubillos Castaño, N. M. (2013). Desempeño del sector Textil - Confección 2008-2012 (Desempeño Sector) (p. 31). Bogotá D.C.: Superintendencia de Sociedades. Recuperado a partir de

von Krogh, G., y Geilinger, N. (2014). Knowledge creation in the eco-system: Research imperatives. En: European Management Journal, Vol.32, No.1, p.155-163.

Wagner, H., y Weitzel, T. (2007). Towards an IT production function. En: Journal of Enterprise Information Management, Vol.20, No.4, p. 380-395.

Wernerfelt, B. (1984). A resource-based view of the firm. En: Strategic Management Journal, Vol.5, núm2, p.171-180.

Winter, S. G. (2003). Understanding dynamic capabilities. En: Strategic Management Journal, Vol.24, No.10, p. 991-995.

Wu, J. (2013). Marketing capabilities, institutional development, and the performance of emerging market firms: A multinational study. En: International Journal of Research in Marketing, Vol.30, No.1, p. 36-45.

Yakhlef, A. (2007). Knowledge transfer as the transformation of context. En: The Journal of High Technology Management Research, Vol.18, No.1, p. 43-57.

Yam, R. C. M., Guan, J. C., Pun, K. F., y Tang, E. P. Y. (2004). An audit of technological innovation capabilities in chinese firms: some empirical findings in Beijing, China. En: Research Policy, Vol.33, No.8, p. 1123-1140.

Yam, R. C. M., Lo, W., Tang, E. P. Y., y Law, A. K. W. (2010). Technological innovation capabilities and firm performance. En: World Academy of Science, Engineering and Technology, Vol.42, p. 1009-1017.

Yang, J. (2005). Knowledge integration and innovation: Securing new product advantage in high technology industry. En: The Journal of High Technology Management Research, Vol.16, No.1, p.121-135.

Yan-Rong, W., y Qiao-Ling, X. (2009). Evaluation of technological innovation capability of enterprises based on perspective of knowledge management. En: 16th International Conference on Industrial Engineering and Engineering Management, p.1967-1972.

Zhang, Q., y Kosaka, M. (2013). SECI model and KIKI model on knowledge creation. En: 2013 10th International Conference on Service Systems and Service Management, p.102-106. 


\section{ANEXO 1. Formulario enviado a expertos}

\begin{tabular}{|c|c|}
\hline \multicolumn{2}{|c|}{$\begin{array}{l}\text { GESTIÓN DE CONOCOCIMIENTO Y CAPACIDADES DE INNOVACIÓN TECNOLÓGICA EN PYMES } \\
\text { DEL SECTOR TEXTIL/ CONFECCIÓN, DISEÑO Y MODA- MEDELLÍN- }\end{array}$} \\
\hline Gestión de conocimiento & Etapas \\
\hline \multirow{2}{*}{$\begin{array}{l}\text { Contempla la conversión de conocimiento tácito (Hace } \\
\text { parte del individuo y no se encuentra codificado); y el } \\
\text { conocimiento explícito (Se encuentra disponible para } \\
\text { todos los miembros de la organización a través de } \\
\text { documentos, manuales y bases de datos). Igualmen- } \\
\text { te, busca transferir el conocimiento desde el punto } \\
\text { de origen, hasta el individuo que lo necesita para el } \\
\text { cumplimiento de sus funciones. }\end{array}$} & $\begin{array}{l}\text { Exploración: Socialización, adquisición, } \\
\text { combinación, internalización, adquisi- } \\
\text { ción }\end{array}$ \\
\hline & $\begin{array}{l}\text { Explotación: Integración, transferencia } \\
\text { y almacenamiento }\end{array}$ \\
\hline Socia & Exteriorización \\
\hline $\begin{array}{l}\text { Compartir experiencias como modelos mentales y ha- } \\
\text { bilidades técnicas. Por ejemplo, capacitación, círculos } \\
\text { de calidad para el desarrollo de nuevos productos. }\end{array}$ & $\begin{array}{l}\text { Conocimiento tácito en explícito; es de- } \\
\text { cir, reformularlo de manera conceptual. } \\
\text { Facilita la creación de nuevos conceptos. }\end{array}$ \\
\hline Combinación & Internalización \\
\hline $\begin{array}{l}\text { Sistematización de los nuevos conceptos con los que } \\
\text { la empresa ya tenía. Por ejemplo, reuniones, docu- } \\
\text { mentos, bases de datos. }\end{array}$ & $\begin{array}{l}\text { Incorporar el conocimiento explícito } \\
\text { en tácito. Por ejemplo, "learning by } \\
\text { doing", que facilita la construcción de } \\
\text { prototipos. }\end{array}$ \\
\hline Adquisición & Integración \\
\hline $\begin{array}{l}\text { El nuevo conocimiento se obtiene a partir de fuentes } \\
\text { internas y externas. Obtención de información de los } \\
\text { clientes, para la satisfacción de sus necesidades. }\end{array}$ & $\begin{array}{l}\text { Integrar el conocimiento especializado } \\
\text { para minimizar los procesos de apren- } \\
\text { dizaje. }\end{array}$ \\
\hline Transferencia & Almacenamiento \\
\hline $\begin{array}{l}\text { Transmisión de conocimiento desde el lugar donde se } \\
\text { produce, hasta el lugar donde debe ser empleado. Por } \\
\text { ejemplo, uso de TIC's y reuniones de trabajo en equipo. }\end{array}$ & $\begin{array}{l}\text { Uso de los depósitos de conocimiento } \\
\text { para no olvidar lo que ya se ha aprendido. } \\
\text { Por ejemplo, bases de datos y uso de } \\
\text { manuales. }\end{array}$ \\
\hline Capacidades de Innovación Tecnológica & Categorías \\
\hline $\begin{array}{l}\text { Son una clase especial de bienes y recursos organiza- } \\
\text { cionales que incluyen tecnología, productos, procesos } \\
\text { y experiencia. De la combinación de estos recursos, } \\
\text { las compañías pueden generar, difundir y utilizar inno- } \\
\text { vaciones que tienen un alto valor económico. }\end{array}$ & $\begin{array}{l}\text { I+D, aprendizaje, manufactura, mar- } \\
\text { keting, direccionamiento estratégico y } \\
\text { explotación de recursos. }\end{array}$ \\
\hline Direccionamiento Estratégico & $\mathrm{I}+\mathrm{D}$ \\
\hline $\begin{array}{l}\text { Identificar DOFA, para asegurar la productividad, el } \\
\text { rendimiento y la armonía. }\end{array}$ & $\begin{array}{l}\text { Integra la estrategia de I+D, con la misión } \\
\text { y visión organizacional. }\end{array}$ \\
\hline
\end{tabular}




\begin{tabular}{|l|l|}
\hline \multicolumn{2}{|c|}{$\begin{array}{l}\text { GESTIÓN DE CONOCOCIMIENTO Y CAPACIDADES DE INNOVACIÓN TECNOLÓGICA EN PYMES } \\
\text { DEL SECTOR TEXTIL/ CONFECCIÓN, DISENO Y MODA- MEDELLíN- }\end{array}$} \\
\hline \multicolumn{1}{|c|}{ Manufactura } & \multicolumn{1}{c|}{ Marketing } \\
\hline $\begin{array}{l}\text { Transforma los resultados de I+D en productos, para } \\
\text { suplir los requerimientos de los clientes. }\end{array}$ & $\begin{array}{l}\text { Habilidad para publicitar y vender pro- } \\
\text { ductos y servicios en un ambiente } \\
\text { competitivo. }\end{array}$ \\
\hline Explotación de recursos & Aprendizaje \\
\hline $\begin{array}{l}\text { Establecimiento de políticas y procedimientos para } \\
\text { apoyar la explotación de recursos. }\end{array}$ & $\begin{array}{l}\text { Habilidad para identificar, asimilar y } \\
\text { aprovechar la información y conoci- } \\
\text { miento. }\end{array}$ \\
\hline
\end{tabular}

\section{GESTIÓN DE CONOCIMIENTO Y CAPACIDADES DE INNOVACIÓN TECNOLÓGICA EN PYMES DEL SECTOR TEXTIL/ CONFECCIÓN, DISEÑO Y MODA- MEDELLÍN-}

\section{OBJETIVO:}

Establecer la relación de causalidad existente entre un conjunto de variables relacionadas con las etapas de gestión de conocimiento (Exploración y explotación), y las capacidades de innovación tecnológica ( $\mathrm{I}+\mathrm{D}$, producción, direccionamiento estratégico y marketing). Dichas variables, se abordarán desde una perspectiva que permita comprender el comportamiento de las Pymes del sector textil en este tema.

INFORMACIÓN GENERAL DEL PARTICIPANTE

\section{Nombre:}

\section{Ocupación:}

\section{Organización:}

\section{Datos de contacto:}

\section{INFORMACIÓN GENERAL DEL SECTOR}

1. ¿Cómo considera usted el posicionamiento actual del sector a nivel nacional e internacional? Favor explique.

2. ¿Cuáles son las principales falencias y limitantes de las pymes del sector? ¿Por qué se presenta?

3. ¿Cuáles son las principales oportunidades de crecimiento de participación en el mercado de las pymes del sector? ¿Cómo lograrlo?

4. ¿Considera importante la relación sector/instituciones universitarias? Favor explique.

5. ¿Considera que la planeación del sector se hace en el corto, mediano o largo plazo? Favor explique. 
Modelo para gestionar el conocimiento en el sector textil de Medellín, empleando dinámica de sistemas

\section{EXPLORACIÓN Y EXPLOTACIÓN DE CONOCIMIENTO, CAPACIDADES DE INNOVACIÓN TECNOLÓGICA}

\begin{tabular}{|c|c|c|c|c|c|c|c|c|c|c|c|c|c|c|c|c|c|c|c|c|}
\hline & \multicolumn{5}{|c|}{ Capacidad I+D } & \multicolumn{5}{|c|}{ Capacidad Producción } & \multicolumn{5}{|c|}{ Capacidad Marketing } & \multicolumn{5}{|c|}{$\begin{array}{c}\text { Capacidad Direccionamien- } \\
\text { to Estratégico }\end{array}$} \\
\hline \multirow[t]{2}{*}{ Círculos de Calidad } & \multicolumn{5}{|c|}{$\begin{array}{c}\text { Identificación de } \\
\text { preferencias }\end{array}$} & \multicolumn{5}{|c|}{$\begin{array}{l}\text { Actualización de maquina- } \\
\text { ria, procesos y equipo }\end{array}$} & \multicolumn{5}{|c|}{ Estimación de la demanda } & \multicolumn{5}{|c|}{ Estrategias de crecimiento } \\
\hline & 1 & 2 & 3 & 4 & 5 & 1 & 2 & 3 & 4 & 5 & 1 & 2 & 3 & 4 & 5 & 1 & 2 & 3 & 4 & 5 \\
\hline \multirow[t]{2}{*}{ Creación de nuevos conceptos } & \multicolumn{5}{|c|}{$\begin{array}{c}\text { Identificación de } \\
\text { preferencias }\end{array}$} & \multicolumn{5}{|c|}{$\begin{array}{l}\text { Actualización de maquina- } \\
\text { ria, procesos y equipo }\end{array}$} & \multicolumn{5}{|c|}{ Estimación de la demanda } & \multicolumn{5}{|c|}{ Estrategias de crecimiento } \\
\hline & 1 & 2 & 3 & 4 & 5 & 1 & 2 & 3 & 4 & 5 & 1 & 2 & 3 & 4 & 5 & 1 & 2 & 3 & 4 & 5 \\
\hline \multirow[t]{2}{*}{ Creación de nuevos productos } & \multicolumn{5}{|c|}{$\begin{array}{c}\text { Identificación de } \\
\text { preferencias }\end{array}$} & \multicolumn{5}{|c|}{$\begin{array}{l}\text { Actualización de maquina- } \\
\text { ria, procesos y equipo }\end{array}$} & \multicolumn{5}{|c|}{ Estimación de la demanda } & \multicolumn{5}{|c|}{ Estrategias de crecimiento } \\
\hline & 1 & 2 & 3 & 4 & 5 & 1 & 2 & 3 & 4 & 5 & 1 & 2 & 3 & 4 & 5 & 1 & 2 & 3 & 4 & 5 \\
\hline Comunicación entre los miembros & \multicolumn{5}{|c|}{$\begin{array}{l}\text { Identificación de } \\
\text { preferencias }\end{array}$} & \multicolumn{5}{|c|}{$\begin{array}{l}\text { Actualización de maquina- } \\
\text { ria, procesos y equipo }\end{array}$} & \multicolumn{5}{|c|}{ Estimación de la demanda } & \multicolumn{5}{|c|}{ Estrategias de crecimiento } \\
\hline & 1 & 2 & 3 & 4 & 5 & 1 & 2 & 3 & 4 & 5 & 1 & 2 & 3 & 4 & 5 & 1 & 2 & 3 & 4 & 5 \\
\hline Visión corporativa & \multicolumn{5}{|c|}{$\begin{array}{c}\text { Identificación de } \\
\text { preferencias }\end{array}$} & \multicolumn{5}{|c|}{$\begin{array}{c}\text { Actualización de maquina- } \\
\text { ria, procesos y equipo }\end{array}$} & \multicolumn{5}{|c|}{ Estimación de la demanda } & \multicolumn{5}{|c|}{ Estrategias de crecimiento } \\
\hline & 1 & 2 & 3 & 4 & 5 & 1 & 2 & 3 & 4 & 5 & 1 & 2 & 3 & 4 & 5 & 1 & 2 & 3 & 4 & 5 \\
\hline Alianzas estratégicas & \multicolumn{5}{|c|}{$\begin{array}{c}\text { Identificación de } \\
\text { preferencias }\end{array}$} & \multicolumn{5}{|c|}{$\begin{array}{c}\text { Actualización de maquina- } \\
\text { ria, procesos y equipo }\end{array}$} & \multicolumn{5}{|c|}{ Estimación de la demanda } & \multicolumn{5}{|c|}{ Estrategias de crecimiento } \\
\hline & 1 & 2 & 3 & 4 & 5 & 1 & 2 & 3 & 4 & 5 & 1 & 2 & 3 & 4 & 5 & 1 & 2 & 3 & 4 & 5 \\
\hline Uso y Acceso a Manuales & \multicolumn{5}{|c|}{$\begin{array}{l}\text { Identificación de } \\
\text { preferencias }\end{array}$} & $\begin{array}{r}\text { Acti } \\
\text { ri }\end{array}$ & & & & & & ació & 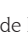 & & nda & Est & teg & de & $\operatorname{cim}$ & ento \\
\hline
\end{tabular}

\begin{tabular}{|c|c|c|c|c|c|c|c|c|c|c|c|c|c|c|c|c|c|c|c|c|}
\hline \multirow[t]{2}{*}{ Adquisición de Conocimiento } & \multicolumn{5}{|c|}{$\begin{array}{l}\text { Creación de Nuevos } \\
\text { Productos }\end{array}$} & \multicolumn{5}{|c|}{ Alianzas Estratégicas } & \multicolumn{5}{|c|}{ Capital } & \multicolumn{5}{|c|}{ Benchmarking } \\
\hline & 1 & 2 & 3 & 4 & 5 & 1 & 2 & 3 & 4 & 5 & 1 & 2 & 3 & 4 & 5 & 1 & 2 & 3 & 4 & 5 \\
\hline Integración de Conocimiento & \multicolumn{5}{|c|}{ Trabajo en Equipo } & \multicolumn{5}{|c|}{$\begin{array}{c}\text { Comunicación entre } \\
\text { miembros }\end{array}$} & \multicolumn{5}{|c|}{ Visión Corporativa } & \multicolumn{5}{|c|}{$\begin{array}{c}\text { Inversión en Capacidades } \\
\text { de I+D }\end{array}$} \\
\hline & 1 & 2 & 3 & 4 & 5 & 1 & 2 & 3 & 4 & 5 & 1 & 2 & 3 & 4 & 5 & 1 & 2 & 3 & 4 & 5 \\
\hline Transferencia de Conocimiento & \multicolumn{5}{|c|}{$\begin{array}{c}\text { Comunicación entre } \\
\text { miembros }\end{array}$} & \multicolumn{5}{|c|}{ Uso de Manuales } & \multicolumn{5}{|c|}{ Visión Corporativa } & \multicolumn{5}{|c|}{$\begin{array}{c}\text { Inversión en Capacidades } \\
\text { de I+D }\end{array}$} \\
\hline & 1 & 2 & 3 & 4 & 5 & 1 & 2 & 3 & 4 & 5 & 1 & 2 & 3 & 4 & 5 & 1 & 2 & 3 & 4 & 5 \\
\hline $\begin{array}{l}\text { Almacenamiento de } \\
\text { Conocimiento }\end{array}$ & \multicolumn{5}{|c|}{$\begin{array}{c}\text { Comunicación entre } \\
\text { miembros }\end{array}$} & \multicolumn{5}{|c|}{ Uso de Manuales } & \multicolumn{5}{|c|}{ Visión Corporativa } & \multicolumn{5}{|c|}{$\begin{array}{c}\text { Inversión en Capacidades } \\
\text { de I+D }\end{array}$} \\
\hline & 1 & 2 & 3 & 4 & 5 & 1 & 2 & 3 & 4 & 5 & 1 & 2 & 3 & 4 & 5 & 1 & 2 & 3 & 4 & 5 \\
\hline
\end{tabular}

1. ¿Qué actividades contribuyen con el desarrollo de nuevos productos, procedimientos; y adquisición de maquinaria y equipo?

2. ¿Considera usted qué la cantidad de líneas de producto y los nuevos procesos organizacionales, desarrollados durante el último año, generan capital adicional a las Pymes?

3. Comentarios adicionales. 


\section{ANEXO 2. \\ Entidades a las cuales están vinculados los actores que participaron en la consulta a expertos}

- Cámara de Comercio de Medellín para Antioquia

- Alcaldía de Medellín

- Inexmoda

- Proantioquia

- $\quad$ CIDET

- Colegiatura Colombiana

- Escuela de Diseño y Mercadeo de Moda Arturo Tejada Cano

- Pontificia Universidad Javeriana 